\title{
Predictores psicológicos de lesión en jóvenes deportistas Psychological Predictors of Injury in Young Athletes
}

\author{
Rosendo Berengüí Gil y Jorge L. Puga \\ Universidad Católica de Murcia (UCAM), España
}

\begin{abstract}
Resumen
En el deporte de competición, una serie de factores fisiológicos, biomecánicos, ambientales y psicológicos interaccionan para provocar la susceptibilidad o un aumento en la vulnerabilidad del deportista a la lesión. Actualmente, es aceptada la importancia de las variables psicológicas en el origen de la lesión y una serie de investigaciones han señalado determinados factores que podrían predecir las lesiones deportivas. El objetivo del estudio es analizar la influencia de las características psicológicas asociadas al rendimiento deportivo, la ansiedad rasgo competitiva y los estados de ánimo en la ocurrencia de lesiones. Los participantes fueron 115 deportistas integrantes de programas de tecnificación, a los cuales fueron administrados el Inventario Psicológico de Ejecución Deportiva (IPED), Sport Competition Anxiety Test (SCAT) y Perfil de Estados de Ánimo (POMS). Posteriormente, se registraron las lesiones durante un período de 10 meses. Los resultados indican que la autoconfianza y la ansiedad rasgo competitiva fueron predictores significativos del número de lesiones. Además, esta relación entre lesiones, ansiedad y autoconfianza se mantuvo cuando se controló el efecto de otras variables. Se discuten estos resultados y se propone una serie de consideraciones a nivel aplicado.
\end{abstract}

Palabras clave: lesiones deportivas, predictores psicológicos, psicología del deporte, tecnificación deportiva, personalidad.

\begin{abstract}
In competitive sports a number of physiological, biomechanical, environmental and psychological factors interact to cause increased susceptibility or vulnerability to injury. We now better understand the importance of psychological variables in the origin of injuries, a number of previous studies have identified certain factors that could predict sports injuries. The aim of this study is to analyze the influence of psychological characteristics associated with athletic performance, competitive trait anxiety and mood in injury occurrence. Participants were 115 athletes, members of specialization programs, who were administered the Psychological Inventory on Athletic Execution (IPED), Sport Competition Anxiety Test (SCAT) and Profile of Mood States (POMS). Subsequent lesions were recorded over a period of 10 months. The results indicate that self-confidence and competitive trait anxiety were significant predictors of the number of injuries. Moreover, this relationship between injuries, anxiety and self-confidence is maintained when the effect of other variables is controlled. These results are discussed and a series of considerations at the applied level are proposed.
\end{abstract}

Keywords: sports injuries; psychological predictors; sports psychology; sports specialization; personality.

Rosendo Berengüí Gil, Facultad de Ciencias Sociales y de la Comunicación, Universidad Católica de Murcia (UCAM), España; Jorge L. Puga, Facultad de Ciencias de la Salud, Universidad Católica de Murcia (UCAM), España.

La correspondencia en relación con este artículo se dirige a Rosendo Berengüí Gil, Grados de Educación y Psicología, Universidad Católica de Murcia, Campus de los Jerónimos, s/n, 30107 Guadalupe (Murcia), Dirección electrónica: rberengui@ucam.edu 
El deporte competitivo requiere altas demandas físicas, técnico-tácticas y psicológicas, y conforme se avanza en edad y nivel de práctica esos requerimientos van en aumento. Una consecuencia lógica de la alta inversión en horas de entrenamientos y exigente competición es el incremento en el riesgo de sufrir una lesión. Su ocurrencia implica un daño físico y suele conllevar una serie de efectos negativos para el deportista, por ejemplo, el cese en el entrenamiento y la competición, la necesidad de atención especializada para su recuperación, además de alteraciones de las rutinas habituales de quien las padece.

Las lesiones no suelen derivar de una única causa, esto es, suelen producirse por una interacción compleja de eventos y múltiples factores de riesgo. Habitualmente, dos son los tipos de factores básicos aceptados: externos o extrínsecos al deportista (como el tipo de deporte, las condiciones ambientales o la ausencia o falta de adherencia a medidas preventivas), e internos o intrínsecos del deportista, concretamente, factores médico-fisiológicos, biomecánicos y psicológicos (como la edad, factores nutricionales o la ejecución de conductas específicas o técnica inadecuada) (Berengüí, Brewer \& Garcés de Los Fayos, 2013; Meeuwisse, Tyreman, Hagel \& Emery, 2007; Wiese-Bjornstal, 2009).

Los factores físicos o fisiológicos en el origen de la lesión son cuantiosos y hasta la fecha han sido los más explorados. Pero, mientras el papel de la preparación mental para el alto rendimiento ha sido ampliamente aceptado y reconocido, la importancia de la influencia de los factores psicológicos sobre el riesgo de lesiones ha sido en numerosas ocasiones subestimada (Junge, 2000).

Desde el campo de la psicología del deporte, se ha trabajado por identificar los antecedentes o los factores de riesgo que motivan la lesión deportiva y se han aportado modelos explicativos de esa relación (Andersen \& Williams, 1988; Buceta, 1996; Junge, 2000; Williams \& Andersen, 1998). La práctica totalidad de modelos sobre los vínculos entre la lesión y los aspectos psicológicos han girado alrededor del estrés como elemento fundamental que influye de muy diferentes formas sobre el individuo, al generar una serie de efectos perjudiciales sobre los componentes físicos o psicológicos del deportista. Pero además, variables de personalidad o recursos de afrontamiento han sido asumidos como posibles precursores de la lesión deportiva. En este contexto, ha pasado más de un cuarto de siglo desde que Andersen \& Williams (1988) plantearan su influyente Modelo de Estrés y Lesión, modelo clásico sobre el que ha girado la gran mayoría de investigaciones en este campo en las últimas décadas.

El Modelo de Estrés y Lesión (Andersen \& Williams, 1988; Williams \& Andersen, 1998) establece que el aumento de los niveles de estrés conlleva un desgaste en la capacidad del individuo para hacer frente adecuadamente a las diferentes situaciones, lo que puede resultar en una lesión. El elemento central y básico del modelo es la respuesta al estrés, la cual supone una relación bidireccional entre la evaluación cognitiva, que el individuo hace de una situación potencialmente estresante y las respuestas fisiológicas y de atención que da al estrés. Así por ejemplo, un deportista puede evaluar cognitivamente las exigencias de la situación de una forma positiva al valorar su adecuación personal para satisfacer esas demandas y poder experimentar la situación como divertida y desafiante. Pero, por el contrario, si la valoración carece de los adecuados o suficientes recursos, se producirá un aumento de la ansiedad y los estados negativos que conlleven la alteración del equilibrio fisiológico y atencional del deportista, lo cual produciría altos niveles de tensión muscular y fallos de concentración.

Además, el modelo propone que esa respuesta al estrés puede verse moderada por determinadas características de personalidad, la historia previa de factores estresantes y los recursos de afrontamiento 
del individuo. Cada uno de esos factores ejercería su influencia de forma aislada o en conjunto con las demás, cuyo resultado agravaría la respuesta al estrés, o por el contrario, su atenuación.

En el modelo original (Andersen \& Williams, 1988) son propuestas cinco variables de personalidad, que moderarían la relación entre estrés y lesión. Dichas variables son dureza, locus de control, ansiedad rasgo competitiva, motivación de logro y sentido de coherencia. A excepción de esta última, todas han sido objeto de investigación en algún grado y se obtienen resultados heterogéneos respecto a su influencia. Posiblemente, la ansiedad rasgo ha sido entre ellas la más analizada, advirtiéndose como altas puntuaciones en ansiedad se ligaron a un mayor número de lesiones o mayor gravedad (Berengüí, 2011; Berengüí \& Garcés de los Fayos, 2010; Berengüí, Garcés de Los Fayos \& Hidalgo-Montesinos, 2013; Blackwell \& McCullagh, 1990; García \& Aragües, 1998; Hanson, McCullagh, P. \& Tonymon., 1992; Johnson \& Ivarsson, 2011; Olmedilla, Andreu, Ortín \& Blas, 2009; Passer \& Seese, 1983; Petrie, 1993; Smith, Ptacek \& Patterson, 2000).

También, al margen de esas cinco variables, otros autores han examinado la relación de otros elementos de la personalidad del deportista o de las características psicológicas asociadas al rendimiento deportivo con las lesiones. Un mayor o menor nivel en dichas características puede conducir al óptimo o deficiente desempeño del deportista en el entrenamiento y las competiciones $\mathrm{y}$, en consecuencia, situarlo en riesgo de sufrir lesiones (Berengüí et al., 2013). Variables tales como el patrón de conducta tipo A (Fields, Delaney \& Hinkle, 1990, Nigorikawa et al., 2003), autoconcepto o autoestima (Kerr \& Minden, 1988, Wittig \& Schurr, 1994), neuroticismo (Berengüí, Garcés de los Fayos \& Almarcha, 2008; Deroche, Stephan, Brewer \& Lescanff, 2007), estabilidad emocional, privacidad y apertura al cambio (Berengüí et al., 2013), autoconfianza (Abenza, Olmedilla, Ortega \& Esparza, 2009; Berengüí, López-Gullón, Garcés de Los Fayos \& Almarcha, 2011, Gimeno, Buceta \& Pérez-Llantada, 2007), concentración (Olmedilla, 2005) o habilidades de afrontamiento (Berengüí et al., 2011, Berengüí et al., 2013, Hanson et al., 1992, Maddison \& Prapavessis, 2005, Ortín, 2008) han mostrado en mayor o menor medida su influencia sobre las lesiones.

Aunque hasta el momento presente son limitados en número, existen estudios que han investigado el rol de las características psicológicas en la predicción de lesiones, si bien la gran mayoría se han centrado en el fútbol y han abordado principalmente el papel del estrés y el apoyo social.

Petrie (1992) encuentra en jugadores de fútbol con bajo apoyo social, que el estrés vital se relaciona positivamente con el número de lesiones graves, tiempo de recuperación, y número de partidos perdidos. Mohad (2006), por su parte, en jugadores de fútbol profesionales encuentra la autoestima, nivel de confianza y la motivación como predictores de lesión.

Ivarsson (2008) distingue cuatro predictores significativos que, en conjunto, explicarían el 23\% de las lesiones: eventos vitales estresantes, la ansiedad rasgo somática, la falta de confianza y el afrontamiento negativo. También, con jugadores de fútbol, Ivarsson \& Johnson (2010) evidencian cuatro predictores: ansiedad somática, ansiedad cognitiva, susceptibilidad al estrés e irritabilidad rasgo. En conjunto, los predictores autoculpa y aceptación podrían explicar el 14,6\% de ocurrencia de lesiones. En jugadores de fútbol de categoría junior, Johnson \& Ivarsson (2011) sugieren cuatro predictores significativos, que juntos podrían explicar el $23 \%$ de que se produzca una lesión. Los principales factores son los eventos vitales estresantes, ansiedad somática, baja confianza y el afrontamiento ineficaz. 
Devantier (2011), en jugadores profesionales de fútbol, indica que la historia previa de lesiones y el afrontamiento de la adversidad fueron los mejores predictores de la aparición de lesiones. Dichos factores explicaron entre el $7 \%$ y el $11 \%$ de la varianza total de la ocurrencia de lesiones y días perdidos debido a una lesión, respectivamente. Además, se encontraron las mismas variables con gran fuerza en la predicción de ocurrencia de lesiones.

Los resultados del análisis de Ivarsson, Johnson \& Podlog (2013) indican que la ansiedad rasgo, el estrés vital negativo y los problemas diarios fueron predictores significativos de lesiones entre los jugadores de fútbol profesionales, lo que representa el $24 \%$ de la varianza, más concretamente, el estrés vital tuvo un efecto indirecto sobre la ocurrencia de lesiones a través de esos acontecimientos diarios.

Respecto a otros deportes, Smith, Smoll \& Ptacek (1990), en deportistas estudiantes de varias modalidades, hallan que el apoyo social funciona en combinación con las habilidades de afrontamiento y que en los deportistas con bajo apoyo social y habilidades de afrontamiento, las diferencias en los acontecimientos vitales negativos representaron casi el 30\% de la varianza de tiempo de baja deportiva.

Galambos, Terry, Moyle \& Locke (2005), a partir de una amplia muestra de 845 deportistas australianos de élite, señalan que el $50 \%$ de la varianza en las puntuaciones de estrés podría predecirse a partir de las puntuaciones de estado de ánimo, especialmente para vigor, depresión y tensión. Las puntuaciones del estado de ánimo y el estrés en conjunto se mostraron significativamente útiles en la predicción de las características de lesiones (estado de la lesión y localización anatómica).

Rubio, Pujals, de la Vega, Aguado y Hernández (2014), con 245 deportistas españoles de diversas disciplinas, hallan que dos estrategias de afrontamiento, calma emocional y tendencia al riesgo, predijeron respectivamente el padecimiento de un menor o mayor número de lesiones, y que la autoeficacia no incidía directamente en el número de lesiones sino que las estrategias de afrontamiento mediaron esa relación. Además, García-Mas, Pujals, Fuster-Parra, Núñez \& Rubio (2014), en una muestra de 297 deportistas de 26 deportes, afirman que una elevada probabilidad de autoeficacia produce dependencia estadística de más probabilidad de lesión deportiva. Entonces, esta variable recibe influencias por antecedentes tanto de variables biomédicas (IMC) como de variables psicológicas (estrés).

En estudios realizados en danza, en un estudio prospectivo de ocho meses, Patterson, Smith, Everett \& Ptacek (1998) encuentran el apoyo social como un factor significativo de vulnerabilidad o protección, y que una combinación de alto estrés vital y bajo apoyo social puede constituir un mayor riesgo de lesión posterior en los bailarines. Los eventos vitales estresantes representaron casi el 50\% de la varianza de lesiones en quienes reportaron bajos niveles de apoyo social en sus vidas. También, Noh, Morris \& Andersen (2005) identifican la ausencia de preocupaciones y la confianza como predictores significativos para la frecuencia de lesiones y esa libertad de preocupación y el estrés negativo como predictores significativos de la duración de la lesión.

El objetivo del presente estudio consiste en analizar la influencia de las características psicológicas asociadas al rendimiento deportivo, la ansiedad rasgo competitiva y los estados de ánimo en la producción de lesiones en deportistas de competición en edades de tecnificación deportiva. 


\section{Método}

\section{Participantes}

Los participantes en el estudio fueron 115 deportistas, 74 hombres $(64,3 \%)$ y 41 mujeres $(35,7 \%)$, practicantes de atletismo (33,9\%), ciclismo (12,2\%), lucha olímpica $(21,7 \%)$, piragüismo $(16,5 \%)$ y taekwondo $(15,7 \%)$. La media de edad fue de 18,72 años $(D T=1,484)$, con un rango de edades entre los 14 y 19 años.

La media de días de entrenamiento semanales es de 5,02 días $(\mathrm{DT}=0,94)$. Los deportistas registraron un promedio de 1,59 lesiones en el período de estudio, con un rango entre una y cinco lesiones. Únicamente cuatro deportistas no registraron lesiones.

Los integrantes de la muestra son competidores de alto nivel competitivo, finalistas en los campeonatos nacionales de sus disciplinas, y forman parte de los programas de tecnificación deportiva de las federaciones de sus respectivos deportes. El 38,7\% han participado, al menos en una ocasión, con sus respectivas selecciones nacionales. Por edades, suele corresponderse con las categorías de competición cadete, juvenil y junior. La tecnificación deportiva se caracteriza por ser un periodo previo al paso al deporte de alto rendimiento o profesionalizado. Los programas buscan un mayor grado de especialización del joven deportista y que él progrese a un nivel más avanzado de entrenamiento, con vistas a su futuro paso al deporte de alta competición.

\section{Instrumentos}

Los datos fueron recopilados mediante la administración de varios instrumentos de evaluación: Inventario Psicológico de Ejecución Deportiva (IPED), Sport Competition Anxiety Test (SCAT), Profile of Mood States (POMS), cuestionario de datos sociodemográficos del deportista y un registro de lesiones.

Inventario Psicológico de Ejecución Deportiva (IPED). El cuestionario es la adaptación del Psychological Performance Inventory (PPI) de Loehr (1986). Se compone por 42 ítems con escala de respuesta tipo Likert de cinco opciones (casi siempre, a menudo, a veces, rara vez, y casi nunca). Se emplea en este trabajo la versión en castellano a partir del trabajo de adaptación y baremación de HernándezMendo (2006) con poblaciones deportivas españolas. Está integrado por siete subescalas:

1. Autoconfianza: evalúa la creencia o el grado de certeza que tiene el deportista respecto a su habilidad para alcanzar el éxito en una determinada tarea y lograr sus objetivos. Implica cogniciones, sentimientos e imágenes positivas acerca de lo que el deportista puede hacer y lograr.

2. Control de afrontamiento negativo: escala referente al control por parte del deportista de emociones negativas, como el miedo, ansiedad, rabia, frustración, etc., así como los esfuerzos cognitivos y conductuales realizados para afrontar las situaciones deportivas adversas.

3. Control de la atención: capacidad de percibir y asimilar de forma apropiada la información que transmiten entrenador y entorno y detección de estímulos significativos de la situación deportiva. Supone, también, la facultad para mantener un enfoque continuo en los aspectos importantes de la tarea e ignorar los irrelevantes. 
4. Control visual e imaginativo: capacidad de controlar situaciones y experiencias a nivel mental, así como de regular el flujo de imágenes mentales en una dirección positiva, constructiva y efectiva.

5. Nivel motivacional: se parte de su importancia como motor del comportamiento. Sus ítems incluyen cuestiones como el grado de compromiso e interés del deportista por su deporte, importancia que le concede o su disposición y afrontamiento respecto al entrenamiento y superación diarios.

6. Control de afrontamiento positivo: es un indicador de la capacidad del deportista para buscar la dinamización a través de emociones positivas, al tiempo que se mantengan controladas las negativas.

7. Control actitudinal: control sobre los hábitos de pensamiento que reflejan el grado en que las propias actitudes personales son consistentes con el rendimiento de alto nivel y dominio sobre la predisposición para la acción.

Las escalas son unidimensionales y presentan valores de fiabilidad entre 0,61 y 0,75 (HernándezMendo, 2006), señalando Hernández-Mendo, Morales-Sánchez y Peñalver (2014), en su trabajo de replicación de las propiedades psicométricas del instrumento, que todos los índices de ajuste se sitúan de forma óptima, y afirmando que la herramienta es adecuada, óptima y parsimoniosa. Se obtienen en este estudio coeficientes (alpha de Cronbach) entre 0.64 en Control de Afrontamiento Positivo y 0.84 en Control Visual e Imaginativo.

Sport Competition Anxiety Test (SCAT). El test de Martens (1977) y Martens, Vealley y Burton (1990) es una medida específica de ansiedad rasgo en el contexto deportivo, que evalúa la tendencia individual a percibir las situaciones competitivas como amenazantes y para responder a esas situaciones con sentimientos de aprensión y tensión. Está integrado por 15 ítems, con tres opciones de respuesta ( $1=$ casi nunca, $2=$ alguna vez y $3=$ a menudo), que permiten obtener una puntuación directa de ansiedad rasgo competitiva. El SCAT ha demostrado aceptables propiedades psicométricas y unos valores de consistencia interna entre 0,95 y 0,97 , y una fiabilidad test-retest en adultos de 0,85 (Martens et al., 1990). Aplicado en el estudio de lesiones, diferentes trabajos obtienen coeficientes de fiabilidad (alpha de Cronbach) de 0.84 (Ortín, 2008) y 0.85 (Berengüí, 2011). En el presente trabajo es de $\alpha=0.85$.

Profile of Mood States (POMS). Instrumento de McNair, Loor y Droppleman (1992), consistente en un autoinforme, cuyo objetivo es evaluar cinco estados de ánimo o estados afectivos: tensión, depresión, cólera, vigor y fatiga. La versión del POMS empleada en este estudio es la forma abreviada adaptada por Fuentes, Balaguer, Melià y García-Merita (1995), conformada por 29 ítems o elementos. Para contestar el cuestionario, se pide al sujeto la valoración, en cuanto al grado en que lo ha experimentado, de cada uno de los adjetivos o estados anímicos, durante un intervalo de tiempo determinado (la semana previa). La escala de respuesta es tipo Likert con cinco opciones $(0=$ nada, $1=$ un poco, $2=$ moderadamente, $3=$ bastante, y $4=$ muchísimo). Los coeficientes de fiabilidad alpha de Cronbach obtenidos son: depresión $=0.84$, cólera $=0.91$, tensión $=0.74$, vigor $=0.80, \mathrm{y}$ fatiga $=0.89$. 
Registro de lesiones. El registro de lesiones se realizó al final de la temporada competitiva. Se empleó un cuestionario utilizado en estudios anteriores (Berengüí, 2011; Berengüí et al., 2013) en el cual los encargados de cumplimentarlo (fisioterapeutas o entrenador) relacionaron las lesiones sufridas durante los diez meses previos, el tiempo (en días) transcurrido desde el inicio hasta la plena recuperación de cada una de las lesiones, así como la causa de la lesión. A nivel operacional, las lesiones registradas fueron definidas como aquellas que resultaron de la participación en un entrenamiento o competición y que implicaron la restricción o el cese completo de la participación del deportista un día o más a partir del día de inicio de la lesión (Hootman, Dick \& Agel, 2007).

\section{Procedimiento}

El primer paso fue la presentación del estudio a los directores técnicos y los responsables de las distintas federaciones deportivas, mediante reuniones en las que se expusieron los objetivos, plan de trabajo y las características básicas de los deportistas que podían participar en el estudio, concretamente, aquellos que formaran parte de sus programas de tecnificación deportiva, hombres y mujeres, y un rango de edad entre 15 y 19 años. Una vez manifestada su aceptación, cada federación propuso un calendario con posibles fechas para poder realizar la toma de datos inicial. La práctica totalidad de sesiones de aplicación de cuestionarios tuvieron lugar en concentraciones técnicas de los diferentes deportes. En diferentes momentos de las concentraciones, se tuvo contacto con los deportistas, a los cuales se les informó del propósito y objetivos del estudio, del tratamiento de privacidad y confidencialidad que gozaría la información por ellos suministrada y se firmó su consentimiento, colaborando los deportistas en el estudio de forma libre y voluntaria. Los menores de edad aportaron, además, un consentimiento firmado de sus padres o tutores legales. Durante la administración de los cuestionarios, los investigadores supervisaron la correcta cumplimentación y ofrecieron ayuda ante diferentes dudas de los atletas.

Respecto a la información de lesiones, esta fue recopilada por los fisioterapeutas y/o entrenadores de los deportistas durante diez meses, desde el comienzo al final de la temporada competitiva. Para la correcta codificación de las lesiones se empleó el sistema OSICS (Orchard Sports Injury Classification System) de Rae \& Orchard (2007).

\section{Análisis de datos}

Se realizaron análisis descriptivos, de correlaciones y análisis de regresión múltiple. Para realizar los cálculos estadísticos se utilizó la versión 3.2.0 de R. Para estimar los coeficientes de regresión estandarizados se usó el paquete QuantPsyc para R, mientras que para estimar la matriz de correlaciones se utilizó el paquete ltm (Rizopoulos, 2006).

\section{Resultados}

En primer lugar, en la Tabla 1 se recogen los estadísticos descriptivos obtenidos para cada una de las variables del estudio. 
Tabla 1

Estadísticos descriptivos de las variables del estudio

\begin{tabular}{|c|c|c|c|c|c|c|c|c|c|c|c|c|c|c|}
\hline & Min. & Max. & $Q 1$ & $Q 2$ & $Q 3$ & $M$ & ETM & $I C_{I}$ & $I C_{S}$ & $D T$ & $S G$ & $K R$ & $k$ & $\alpha$ \\
\hline Ls. & 0 & 5 & 1 & 1 & 2 & 1.59 & 0.09 & 1.42 & 1.76 & 0.93 & 1.61 & 1.87 & & \\
\hline$A C$ & 9 & 30 & 19 & 23 & 26 & 22.37 & 0.45 & 21.48 & 23.25 & 4.82 & -0.61 & -0.19 & 6 & .80 \\
\hline$C A N$ & 10 & 29 & 16 & 20 & 23 & 19.73 & 0.41 & 18.93 & 20.53 & 4.35 & -0.18 & -0.69 & 6 & .78 \\
\hline $\boldsymbol{C A T}$ & 12 & 30 & 18.5 & 22 & 24 & 21.21 & 0.38 & 20.46 & 21.95 & 4.03 & -0.42 & -0.43 & 6 & .77 \\
\hline$C V I$ & 9 & 30 & 21 & 25 & 28 & 23.79 & 0.45 & 22.91 & 24.67 & 4.78 & -0.97 & 0.60 & 6 & .84 \\
\hline$N M$ & 10 & 30 & 22 & 25 & 27 & 24.53 & 0.34 & 23.86 & 25.20 & 3.61 & -0.76 & 1.05 & 6 & .79 \\
\hline$C A P$ & 13 & 30 & 21.5 & 24 & 26 & 23.77 & 0.32 & 23.13 & 24.42 & 3.48 & -0.34 & -0.13 & 6 & .64 \\
\hline$A C T$ & 11 & 30 & 20.5 & 23 & 25.5 & 22.88 & 0.36 & 22.17 & 23.59 & 3.85 & -0.45 & -0.12 & 6 & .69 \\
\hline$S C A T$ & 10 & 30 & 19 & 23 & 26.5 & 22.40 & 0.46 & 21.49 & 23.31 & 4.94 & -0.41 & -0.92 & 10 & .85 \\
\hline$D$ & 0 & 21 & 0 & 2 & 4 & 3.36 & 0.39 & 2.59 & 4.12 & 4.14 & 2.07 & 4.9 & 6 & .84 \\
\hline$C$ & 0 & 22 & 1 & 4 & 7 & 5.45 & 0.5 & 4.46 & 6.44 & 5.35 & 1.23 & 0.91 & 6 & .91 \\
\hline$T$ & 0 & 18 & 5 & 8 & 12 & 8.38 & 0.42 & 7.54 & 9.22 & 4.55 & 0.15 & -0.92 & 6 & .74 \\
\hline $\boldsymbol{V}$ & 6 & 24 & 15 & 17 & 21 & 17.5 & 0.36 & 16.8 & 18.3 & 3.9 & -0.26 & -0.59 & 6 & .80 \\
\hline$F$ & 0 & 19 & 2 & 4 & 8 & 5.31 & 0.41 & 4.51 & 6.12 & 4.36 & 0.9 & 0.46 & 5 & .89 \\
\hline
\end{tabular}

Nota. Min.: mínimo, Max.: máximo, Q1: primer cuartil, Q2: segundo cuartil, Q3: tercer cuartil, M: media, ETM: error típico de la media, $I C_{I}$ : límite inferior del intervalo de confianza inferior para la media al $95 \%, I C_{S}$ : límite superior intervalo de confianza superior para la media al 95\%,Var: varianza, DT: desviación típica, $S G$ : sesgo, KR: kurtosis, $k$ : número de ítems, $\alpha$ : índice de consistencia interna de Cronbach, Ls.: lesiones, $A C$ : Autoconfianza, CAN: Control de Afrontamiento Negativo, CAT: Control de la Atención, CVI: Control Visual e Imaginativo, NM: Nivel Motivacional, CAP: Control de Afrontamiento Positivo, $A C T$ : Control Actitudinal, SCAT: Ansiedad rasgo competitiva, D: Depresión, C: Cólera, T: Tensión, $V$ : Vigor, $F$ : Fatiga.

En la Tabla 2 aparece la matriz de correlaciones entre el número de lesiones y las distintas variables analizadas. De entre las escalas que componen el Inventario Psicológico de Ejecución Deportiva, la variable que más relación mostró con el número de lesiones es autoconfianza $(r=-.58, p<.001$, contraste bilateral), mientras que la que menor asociación muestra es nivel motivacional $(r=-.32, p<$ .001 , contraste bilateral). La variable control visual e imaginativo fue la única del grupo que no se mostró asociada al número de lesiones $(r=-.14, p=.13$, contraste bilateral). Tanto la puntuación en control de afrontamiento negativo $(r=-.45, p<.001$, contraste bilateral), control atencional $(r=-.43, p<.001$, contraste bilateral), control de afrontamiento positivo $(r=-.36, p<.001$, contraste bilateral) y control actitudinal ( $r=-.35, p<.001$, contraste bilateral) estuvieron, también, inversamente relacionadas con el número de lesiones; es decir, que a mayor puntuación en cada una de estas variables menor número de lesiones se apreció en los deportistas.

Todas las puntuaciones derivadas del perfil de Estados de animos mostraron una relación positiva con el número de lesiones, al igual que la puntuación en ansiedad rasgo, medida mediante el SCAT ( $r=$ $.43, p<.001$, contraste bilateral), excepto la puntuación en Vigor, que tuvo el signo contrario $(r=-.27, p=$ 0.004 , contraste bilateral). De las escalas que componen el POMS, la que mayor relación positiva mostró 
con el número de lesiones es fatiga $(r=.38, p<.001$, contraste bilateral) y la que menos fue depresión $(r=$ $.17, p=.072$, contraste bilateral). Por su parte, la correlación observada entre el número de lesiones y la escala tensión fue de .24 ( $p<.001$, contraste bilateral), mientras que para la escala cólera fue de .36 ( $p<$ .001 , contraste bilateral).

Tabla 2

Matriz de correlaciones de las variables del estudio

\begin{tabular}{|c|c|c|c|c|c|c|c|c|c|}
\hline & Lesiones & $A C$ & $C A N$ & $C A T$ & CVI & $N M$ & $C A P$ & $A C T$ & SCAT \\
\hline Lesiones & & $-.58 * *$ & $-.45 * *$ & $-.43^{* *}$ & -.14 & $-.32 * *$ & $-.36^{* *}$ & $-.35 * *$ & $.43 * *$ \\
\hline$A C$ & & & $.68 * *$ & $.55 * *$ & $.37 * *$ & $.55 * *$ & $.64 * *$ & $.76^{* *}$ & $-.44 * *$ \\
\hline$C A N$ & & & & $.60 * *$ & $.27 * *$ & $.47 * *$ & $.56^{* *}$ & $.59 * *$ & $-.51 * *$ \\
\hline$C A T$ & & & & & $.22 *$ & $.56 * *$ & $.53 * *$ & $.58 * *$ & $-.47 * *$ \\
\hline$C V I$ & & & & & & $.41 * *$ & $.36^{* *}$ & $.39 * *$ & $-.32 * *$ \\
\hline$N M$ & & & & & & & $.61^{* *}$ & $.62 * *$ & $-.27 * *$ \\
\hline$C A P$ & & & & & & & & $.75^{* *}$ & $-.32 * *$ \\
\hline$A C T$ & & & & & & & & & $-.34 * *$ \\
\hline
\end{tabular}

Nota. AC: Autoconfianza, CAN: Control de Afrontamiento Negativo, CAT: Control de la Atención, CVI: Control Visual e Imaginativo, NM: Nivel Motivacional, CAP: Control de Afrontamiento Positivo, ACT: Control Actitudinal, SCAT: Ansiedad rasgo competitiva.

${ }^{*} p<.05, * * p<.01$, todos los contrastes son bilaterales.

En la Tabla 3 aparecen resumidos los resultados de los ocho modelos de regresión lineal estimados para estudiar la relación condicional que se establece entre el número de lesiones y las variables del inventario psicológico de ejecución deportiva. Las puntuaciones que se derivan del perfil de estados de ánimo no mostraron ser susceptibles de ser analizadas bajo esta estrategia, ya que no se observaron patrones de correlación estadísticamente significativa cuando se controló el efecto del resto de las variables. Dicho de otro modo, las únicas correlaciones significativas observadas fueron las bivariadas y cuando se controló el efecto de cualquier variable esta relación desapareció. Cada modelo añade la variable que más se asocia con el número de lesiones sin contar las precedentes. El primer modelo, que explica un $33 \%$ de la variabilidad de los datos, solo incluye la puntuación de autoconfianza dado que es la que más relación ha mostrado tener con el número de lesiones. Como se puede apreciar en la Tabla 2, la variable autoconfianza muestra una relación estadísticamente significativa de tipo negativo con la ocurrencia de lesiones en los deportistas que se mantiene constante cuando se controla el efecto de todas las variables del estudio. Aunque esta relación tiende a decrecer sensiblemente cuando incluimos control de afrontamiento negativo y la puntuación en ansiedad rasgo, únicamente la inclusión de esta segunda variable muestra una reducción significativa de la magnitud de asociación que se establece entre ambas variables.

La contribución conjunta de la autoconfianza y la ansiedad rasgo se mantiene relativamente estable hasta que la puntuación de control actitudinal es incluida en el sexto modelo, el cual muestra un incremento significativo en el porcentaje de varianza, que es capaz de explicar en comparación con los modelos previos (38\%). En este momento, la contribución de ansiedad rasgo deja de ser relevante para 
explicar el número de lesiones en los deportistas y, aunque vuelve a formar parte significativa del modelo cuando todas las variables han sido incluidas, esta contribución no mejora sustancialmente la variabilidad explicada por los modelos anteriores.

Tabla 3

Parámetros asociados a los modelos de regresión lineal destinados a valorar la fuerza de asociación condicional entre el número de lesiones y las puntuaciones en las escalas

\begin{tabular}{|c|c|c|c|c|c|c|c|c|c|c|c|c|c|}
\hline & & $A C$ & $C A N$ & $S C A T$ & $C A T$ & $C A P$ & $A C T$ & $N M$ & $C V I$ & $R^{2}$ & $D R^{2}$ & $F$ & $p$ \\
\hline \multirow{2}{*}{ M1 } & $b$ & -0.58 & & & & & & & & \multirow{2}{*}{.33} & & & \\
\hline & $t$ & $-7.56^{\#}$ & & & & & & & & & & & \\
\hline \multirow{2}{*}{$M 2$} & $b$ & -0.5 & -0.11 & & & & & & & \multirow{2}{*}{.33} & \multirow[b]{2}{*}{.00} & \multirow[b]{2}{*}{1.13} & \multirow[b]{2}{*}{.29} \\
\hline & $t$ & $-4.85^{\#}$ & -1.07 & & & & & & & & & & \\
\hline \multirow{2}{*}{ M3 } & $b$ & -0.47 & -0.03 & 0.21 & & & & & & \multirow{2}{*}{.36} & \multirow{2}{*}{.03} & \multirow{2}{*}{5.45} & \multirow{2}{*}{.02} \\
\hline & $t$ & $-4.55^{\#}$ & -0.27 & $2.34^{*}$ & & & & & & & & & \\
\hline \multirow{2}{*}{ M4 } & $b$ & -0.45 & 0.00 & 0.19 & -0.09 & & & & & \multirow{2}{*}{.36} & \multirow{2}{*}{.00} & \multirow{2}{*}{0.86} & \multirow{2}{*}{.36} \\
\hline & $t$ & $-4.22^{\#}$ & 0.02 & $2.09^{*}$ & -0.93 & & & & & & & & \\
\hline \multirow{2}{*}{ M5 } & $b$ & -0.48 & -0.01 & 0.19 & -0.11 & 0.07 & & & & \multirow{2}{*}{.35} & \multirow{2}{*}{.00} & \multirow{2}{*}{0.49} & \multirow{2}{*}{.49} \\
\hline & $t$ & $-4.15^{\#}$ & -0.07 & $2.04^{*}$ & -1.06 & 0.7 & & & & & & & \\
\hline \multirow{2}{*}{ M6 } & $b$ & -0.62 & -0.01 & 0.17 & -0.15 & -0.06 & 0.32 & & & \multirow{2}{*}{.38} & \multirow{2}{*}{.03} & \multirow{2}{*}{5.41} & \multirow{2}{*}{.02} \\
\hline & $t$ & $-4.83^{\#}$ & -0.11 & 1.85 & -1.51 & -0.5 & $2.33^{*}$ & & & & & & \\
\hline \multirow{2}{*}{ M7 } & $b$ & -0.62 & -0.01 & 0.17 & -0.15 & -0.05 & 0.32 & -0.02 & & \multirow{2}{*}{.37} & \multirow{2}{*}{-.01} & \multirow{2}{*}{0.02} & \multirow{2}{*}{.88} \\
\hline & $t$ & $-4.78^{\#}$ & -0.11 & 1.84 & -1.41 & -0.45 & $2.31^{*}$ & -0.15 & & & & & \\
\hline \multirow{2}{*}{ M8 } & $b$ & -0.63 & 0.00 & 0.19 & -0.13 & -0.06 & 0.3 & -0.05 & 0.1 & \multirow{2}{*}{.38} & 00 & 148 & 23 \\
\hline & $t$ & $-4.86^{\#}$ & -0.03 & $2.08^{*}$ & -1.19 & -0.51 & $2.18^{*}$ & -0.44 & 1.22 & & .00 & 1.40 & . \\
\hline
\end{tabular}

Nota. $M n$ : modelo enésimo, $b$ : coeficiente de regresión lineal estandarizado, $A C$ : Autoconfianza, CAN: Control de Afrontamiento Negativo, CAT: Control de la Atención, CVI: Control Visual e Imaginativo, NM: Nivel Motivacional, CAP: Control de Afrontamiento Positivo, ACT: Control Actitudinal, SCAT: Ansiedad rasgo competitiva, \#: $p<.001,{ }^{*}: p<.05, R^{2}$ : erre cuadrado ajustada en función del número de variables, $\Delta R^{2}$ : incremento en erre cuadrado en comparación con el modelo $n$ - $1, F$ : estadístico de contraste para evaluar el incremento producido en la bondad de ajuste en relación con el modelo $n$ - $1, p$ : p-valor relativo al incremento de la bondad de ajuste.

\section{Discusión}

El objetivo de este estudio ha sido examinar la contribución de las características psicológicas asociadas al rendimiento deportivo, la ansiedad rasgo competitiva y los estados de ánimo en la predicción del número de lesiones deportivas. Los resultados muestran que existen factores psicológicos que pueden predecir el número de lesiones, concretamente, la autoconfianza y la ansiedad rasgo competitiva, así como el control de actitudes en menor grado. 
La autoconfianza tiene una relación consistente y estable con el número de lesiones, pese a controlarse el efecto que surte el resto de variables utilizadas en el estudio. Esta relación es de tipo negativo de proporcionalidad inversa. Esto es, cuanto mayor es la puntuación en autoconfianza, menos lesiones sufren los deportistas.

La autoconfianza es la creencia o el grado de certeza en que se dispone de los recursos internos o habilidades particulares, para lograr el éxito, en concreto y en este ámbito, en el deporte (Vealey, 2009) e implica cogniciones, sentimientos e imágenes positivas acerca de lo que el deportista puede hacer y lograr. Esa certeza en la propia capacidad es determinada por la experiencia, los estados fisiológicos y emocionales (Hernández-Mendo, 2006), e indica Feltz (2007) que una de las conclusiones de investigación más consistentes es la correlación significativa existente entre la autoconfianza y el rendimiento deportivo de alto nivel. Habitualmente, niveles altos de confianza han sido asociados con afecto positivo y, por el contrario, niveles inferiores se asocian con ansiedad, depresión e insatisfacción (Martens et al., 1990; Vealey, Hayashi, Garner-Holman \& Giacobbi, 1998).

Estudios previos aportan resultados que siguen la línea de los obtenidos aquí en cuanto al esencial peso de la autoconfianza. Las investigaciones en futbolistas de Ivarsson (2008), Johnson \& Ivarsson (2011), Mohad (2006), Noh et al. (2005) en bailarines, muestran que bajos niveles de confianza son predictores significativos de lesión. Además, otros estudios obtienen datos que señalan la importancia de la autoconfianza frente a las lesiones. Abenza et al. (2009) obtienen que valores medios y bajos en la variable están asociados a un mayor riesgo de padecer lesiones, Berengüí et al. (2011) hallan correlación significativa negativa entre autoconfianza y el número de lesiones y Gimeno et al. (2007) concluyen que la baja confianza está asociada a una incidencia mayor de lesiones por sobrecarga.

Estamos de acuerdo con Berengüí (2011) al considerar que la evaluación positiva del deportista de sus propias capacidades para ejecutar de forma exitosa, actuar con seguridad y mantener estable esa creencia en la capacidad personal, son condiciones imprescindibles para el óptimo rendimiento, el progreso deportivo y, en este sentido, para llevar a cabo realizaciones técnicas y tácticas adecuadas y adaptadas a la situación deportiva, que disminuyan consecuentemente el riesgo de lesión.

También, la variable ansiedad rasgo competitiva contribuye junto con la autoconfianza a explicar el número de lesiones que experimentan los deportistas. Sin embargo, en este caso, la relación es directamente proporcional o positiva. Esto significa que a mayores puntuaciones en ansiedad rasgo competitiva, mayor número de lesiones en los deportistas.

La ansiedad competitiva es un rasgo de personalidad relativamente estable, y da cuenta de las diferencias individuales en la tendencia para percibir una amplia gama de situaciones o circunstancias de competición como amenazantes, y a responder a las mismas con estados de ansiedad de intensidad y magnitud desproporcionadas con respecto a la demanda (Marten, 1977). La ansiedad, generalmente, resulta debilitante sobre el rendimiento deportivo porque, dependiendo de la naturaleza de la tarea implicada, obstaculiza la mayoría de formas de rendimiento deportivo, y es especialmente disruptiva en habilidades motoras finas (Smith, Smoll \& Wiechman, 1998).

El papel de la ansiedad es uno de los hallazgos más sólidos en este campo, ya que existen multitud de ejemplos en los que la ansiedad rasgo competitiva se asocia a un mayor número de lesiones o mayor gravedad de las mismas (Berengüí et al., 2013; Blackwell \& McCullagh, 1990; Hanson et al., 
1992; Johnson \& Ivarsson, 2011; Olmedilla et al., 2009; Passer \& Seese, 1983; Petrie, 1993; Smith et al., 2000). En los estudios de Ivarsson (2008), Ivarsson \& Johnson (2010), Ivarsson et al. (2013), y Johnson \& Ivarsson (2011), la ansiedad se mostró como un importante predictor de las lesiones.

Compartimos los planteamientos de Williams \& Andersen (1998), puesto creemos que los altos niveles de ansiedad pueden desequilibrar las apropiadas condiciones fisiológicas y de concentración necesarias para una correcta ejecución, y llevar ligado inevitablemente un aumento en la vulnerabilidad a la lesión. Además, en los deportistas con esa tendencia individual a percibir las situaciones competitivas como amenazantes y a responder a esas situaciones con sentimientos de aprensión y tensión, como definen los autores del SCAT (Martens, 1977; Martens et al., 1990), es más que probable que puedan albergar estados afectivos y de activación física y psicológica que sitúen su organismo en una posición de riesgo para padecer las lesiones, por la ausencia del pertinente ajuste global que demanda la ejecución deportiva.

La variable control actitudinal también, parece contribuir positivamente a explicar el número de lesiones de los deportistas cuando se controlan el resto de las variables del estudio. Ese control de actitudes refleja el hábito de pensamiento del deportista al señalar el grado en que sus actitudes personales son coherentes con los resultados de alto nivel, lo cual afecta la actitud correcta a las demás variables psicológicas (Loehr, 1986). A pesar de no localizar estudios que confirmen la relación de esta variable con las lesiones deportivas, podemos advertir la importancia que puede tener en su ocurrencia. El control actitudinal supone para Hernández-Mendo (2006) el dominio sobre la predisposición para la acción y para la clasificación de los objetos, personas y conductas del entorno del individuo, y sobre el grado de reacción ante estos y su consistencia evaluativa. Un adecuado control de actitudes podría constituir una disposición positiva del deportista hacia su práctica y lo mantendría inmerso en una dinámica productiva que le conduzca al control de estresores y emociones negativas.

Sin embargo, debemos señalar que en nuestros análisis, cuando se estima la correlación bivariada entre las lesiones y control actitudinal tenemos un valor negativo, pero cuando se controla el efecto del resto de variables la relación pasa a ser positiva, quizá por el posible efecto de variables confusoras. Por ello, se precisan más estudios al respecto con otros métodos o análisis para indagar sobre la relación que se establece entre estas variables. En este sentido, respecto a los estados de ánimo, en el presente estudio no muestran un efecto significativo. Galambos et al. (2005) hallaban determinados estados como útiles en la predicción de características de lesiones (estado de la lesión y localización anatómica), las cuales no son asumidas en este estudio. Al igual que con la anterior variable, consideramos necesario profundizar en su análisis con diferentes muestras, instrumentos y la asunción de más variables que aporten mayor información sobre el peso de los estados de ánimo en la lesión.

Desde el punto de vista metodológico, estos resultados han de ser tomados con cautela y únicamente de modo descriptivo. En primer lugar, el tamaño de la muestra es moderado y ello podría afectar a la representatividad de los resultados obtenidos. Por otra parte, la validez de los resultados encontrados con base en la técnica de análisis de datos empleada, podría verse amenazada debido al sesgo y la curtosis de las lesiones deportivas, así como por la presencia de problemas de multicolinealidad. Para futuras investigaciones sugerimos que se utilicen modelos multivariados de red bayesiana (véase, por ejemplo, López, García, De la Fuente \& De la Fuente, 2007) del mismo modo a como han hecho recientemente otros autores (García-Mas et al., 2014; García-Mas et al., 2015) para analizar variables relacionadas con el rendimiento deportivo. 
Del mismo modo, respecto a futuras investigaciones, compartimos el punto de vista de Johnson, Tranaeus \& Ivarsson (2014) en cuanto a la necesidad de seguir profundizando en el estudio en este ámbito y considerar diferentes muestras y modalidades deportivas, el empleo de estadísticos que verifiquen las complejas interacciones y las diferencias individuales, así como la propuesta de diseños dirigidos a la intervención en la prevención de lesiones.

Consideramos los resultados aquí obtenidos de especial relevancia para el campo profesional aplicado. Las características de los deportistas, que conformaron la muestra, pertenecientes a programas de tecnificación deportiva, hacen que desarrollen un deporte de alto nivel, previo al profesionalismo o el alto rendimiento deportivo. Por ello, las exigencias físicas, técnico-tácticas y psicológicas requeridas suponen un drástico cambio a nivel cuantitativo y cualitativo respecto a edades previas, en las que suele predominar un deporte más lúdico y un entrenamiento menos intensivo. El riesgo de sufrir una lesión lógicamente aumenta. Tanto el psicólogo deportivo, como el entrenador y demás figuras alrededor del deportista, deben ser sensibles a esos cambios y ajustar su trabajo a la realidad del joven atleta.

La prevención de lesiones, habitualmente, ha buscado proporcionar seguridad al deportista mediante equipos protectores, instalaciones seguras, y una más óptima preparación física (Brewer, 2009), pero a la vista de los resultados aportados por la investigación en psicología del deporte, resulta imprescindible la necesidad de trabajar sobre los factores psicológicos y los recursos de afrontamiento del deportista para hacerlo menos vulnerable a la lesión.

Los datos generados por este estudio indican que aquellos jóvenes deportistas con menores niveles de confianza en sí mismos y mayores de ansiedad, presentan un mayor número de lesiones. Detectadas esas carencias, el siguiente paso debe ser poner en práctica intervenciones destinadas al entrenamiento de esas variables con el objetivo de minimizar al máximo el riesgo de aparición de lesiones y, en segundo término, evitar el negativo impacto que estas tienen sobre el rendimiento deportivo.

En este campo es posible trabajar en diversas direcciones y con variada metodología. Técnicas de intervención como, por ejemplo, el entrenamiento en biorretroalimentación, en respiración y relajación, la visualización, y multitud de técnicas para el control de la conducta o de aspectos cognitivos, o el fortalecimiento de la efectiva comunicación entre deportista y entrenador, han demostrado su sobrada eficacia orientados al rendimiento efectivo del deportista. También, determinadas intervenciones psicológicas, compuestas por una o varias de esas técnicas, se han mostrado valiosas a la hora de dotar al deportista de recursos de afrontamiento para el control del estrés y la ansiedad, así como en la reducción de lesiones, en mayor o menor medida, con descensos en las tasas de lesión como resultado de la formación en técnicas cognitivas, biofeedback, relajación, visualización guiada, o control atencional, entre otras (Williams \& Andersen, 2007).

Asimismo, un buen número de estrategias pueden contribuir a construir una ajustada autoconfianza del deportista. Integrar elementos como el adecuado establecimiento de objetivos, promover un hábito de pensamiento positivo, el fomento de expectativas adecuadas y realistas sobre el rendimiento, o aportar continuo refuerzo y realimentación al progreso del deportista, puede conducir a que genere sentimientos de disfrute y realización con su práctica y evitando igualmente mayor presión de la que ya aporta la competición por sí misma. 


\section{Referencias}

Abenza, L., Olmedilla, A., Ortega, E. \& Esparza, F. (2009). Lesiones y factores psicológicos en futbolistas juveniles. Archivos de Medicina del Deporte, 132, 280-288.

Andersen, M. B. \& Williams, J. M. (1988). A model of stress and athletic in-jury: Prediction and prevention. Journal of Sport and Exercise Psychology, 104(3), 294-306.

Berengüí, R. (2011). Personalidad, características psicológicas y lesiones en el deporte de competición: Análisis de relaciones e incidencia en jóvenes deportistas (Tesis Doctoral). Universidad de Murcia, Murcia.

Berengüí, R., Brewer, B. W. \& Garcés de Los Fayos, E. J. (2013). Aspectos psicológicos en las lesiones deportivas. En F. Arbinaga y E. Cantón, Psicología del Deporte y la Salud: Una relación compleja, (pp. 197-244). Madrid: EOS.

Berengüí, R. \& Garcés de Los Fayos, E. J. (2010). Ansiedad y lesiones deportivas: Estudio correlacional. Congreso Nacional de Psicología de la Actividad Física y el Deporte. Madrid: SEPD.

Berengüí, R., Garcés de Los Fayos, E. J. \& Hidalgo-Montesinos, M. D. (2013). Características psicológicas asociadas a la incidencia de lesiones en deportistas de modalidades individuales. Anales de Psicología, 29(3), 674-684.

Berengüí, R., López-Gullón, J. M., Garcés de Los Fayos, E. J. \& Almarcha, J. (2011). Factores psicológicos y lesiones deportivas en lucha olímpica y taekwondo. E-balonmano.com: Revista de Ciencias del Deporte, 7(Supl.), 91-98.

Blackwell, B. \& McCullagh, P. (1990). The relationship of athletic injury to life stress, competitive anxiety and coping resources. Athletic Training, 25(1), 23-27.

Brewer, B.W. (2009). Sport Psychology. Handbook of Sports Medicine and Science. Oxford: John Wiley \& Sons.

Buceta, J.M. (1996). Psicología y lesiones deportivas: prevención y recuperación. Madrid: Dykinson.

Deroche, T., Stephan, Y., Brewer, B. W. \& Lescanff, C. (2007). Predictors of perceived susceptibility to sportrelated injury. Personality and Individual Differences, 43(8), 2218-2228.

Devantier, C. (2011). Psychological Predictors of Injury among Professional Soccer Players. Sport Science Review, 20(5-6), 5-36.

Feltz, D. L. (2007). Self-confidence and sports performance. En D. Smith y M. Bar-Eli (Eds.), Essential readings in sport and exercise psychology. Champaign: Human Kinetics.

Fields, K. B., Delaney, M. \& Hinkle, J. C. (1990). A prospective study of type A behavior and running injuries. Journal of Family Practice, 30(4), 425-429.

Fuentes, I., Balaguer, I., Melià, J. L. \& García-Merita, M. L. (1995). Forma abreviada del perfil de estados de ánimo (POMS). V Congreso Nacional de Psicología de la Actividad Física y el Deporte, Universitat de Valencia, Valencia.

Galambos, S. A., Terry, P. C., Moyle, G. M. \& Locke, S. A. (2005). Psychological predictors of injury among elite athletes. British Journal of Sports Medicine, 39, 351-354. 
García, A. P. \& Aragües G. M. (1998). Sport injuries and level of anxiety in soccer players. Medicina Clínica, $111(2), 45-48$

García-Mas, A., Fuster-Parra, P., Ponseti, F. J., Palou, P., Olmedilla, A. \& Cruz, J. (2015). Análisis bayesiano de la motivación, el clima motivacional y la ansiedad en jóvenes jugadores de equipo. Anales de Psicología, 31, 355-366.

García-Mas, A., Pujals, C., Fuster-Parra, P., Núñez, A. \& Rubio, V. J. (2014). Determinación de las variables psicológicas y deportivas relevantes a las lesiones deportivas: Un análisis bayesiano. Revista de Psicología del Deporte, 23(2), 423-429.

Gimeno, F., Buceta, J. M. \& Pérez-Llantada, M. C. (2007). Influencia de las variables psicológicas en el deporte de competición: evaluación mediante el cuestionario Características psicológicas relacionadas con el rendimiento deportivo. Psicothema, 19(4), 667-672.

Hanson, S. J., McCullagh, P. \& Tonymon, P. (1992). The relationship of personality characteristics, life-stress, and coping resources to athletic injury. Journal of Sport and Exercise Psychology, 14(3), 262-272.

Hernández-Mendo, A. (2006). Un cuestionario para la evaluación psicológica de la ejecución deportiva: Estudio complementario entre TCT y TRI. Revista de Psicología del Deporte, 15(1), 71-93.

Hernández-Mendo, A., Morales-Sánchez, V. \& Peñalver, I. (2014). Replicación de las propiedades psicométricas del Inventario Psicológico de Ejecución Deportiva. Revista de Psicología del Deporte, 23(2), 311-324.

Hootman, J. M., Dick, R. \& Agel, J. (2007). Epidemiology of collegiate injuries for 15 sports: Summary and recommendations for injury prevention initiatives. Journal of Athletic Training, 42(2), 311-319.

Ivarsson, A. (2008). Psychological Predictors of Sport Injuries among Soccer Players. Halmstad: University of Halmstad.

Ivarsson, A. \& Johnson, U. (2010). Psychological factors as predictors of injuries among senior soccer players. A prospective study. Journal of Sports Science and Medicine, 9, 347-352.

Ivarsson, A., Johnson, U. \& Podlog, L. (2013). Psychological predictors of injury occurrence: A prospective investigation of professional swedish soccer players. Journal of Sport Rehabilitation, 22, 19-26.

Johnson, U. \& Ivarsson, A. (2011). Psychological predictors of sport injuries among junior soccer players. Scandinavian Journal of Medicine and Science in Sport, 21, 129-136.

Johnson, U., Tranaeus, U. \& Ivarsson, A. (2014). Current status and future challenges in psychological research of sport injury prediction and prevention: A methodological perspective. Revista de Psicología del Deporte, 23(2), 401-409.

Junge, A. (2000). The influence of psychological factors on sports injuries: Review of the literature. The American Journal of Sports Medicine, 28 (5), S10-15.

Kerr, G. \& Minden, H. (1988). Psychological factors related to the occurrence of athletic injuries. Journal of Sport and Exercise Psychology, 10, 167-173.

Loehr, J. E. (1986). Mental toughness training for sports: Achieving athletic excellence. Lexington: Stephen Greene Press. 
López, J., García, J., De la Fuente, L. \& De la Fuente, E. I. (2007). Las redes bayesianas como herramienta de modelado en psicología. Anales de Psicología, 23, 577-581.

Maddison, R. \& Prapavessis, H. (2005). A psychological approach to the prediction and prevention of athletic injury. Journal of Sport and Excercise Psychology, 27, 289-310.

Martens, R. (1977). Sport Competition Anxiety Test. Champaign: Human Kinetics.

Martens, R., Vealley, R. \& Burton, D. (1990). Competitive anxiety in sport. Champaign: Human Kinetics.

McNair, D. M., Lorr, M. \& Droppleman, L. F. (1992). Manual for the Profile of Mood States. North Tonawanda: MultiHealth System Inc.

Meeuwisse, W. H., Tyreman, H., Hagel, B. \& Emery, C. (2007). A dynamic model of etiology in sport injury: the recursive nature of risk and causation. Clinical Journal of Sports Medicine, 17, 215-219.

Mohad, A. B. (2006). Psychological predictors of injury among Malaysian professional football players (Tesis Doctoral). Universiti Sains Malaysia, Gegulor.

Nigorikawa, T., Oishi, K., Yasukawa, M., Kamimura, M., Murayama, M. \& Tanaka, N. (2003). Type A behavior pattern and sports injuries. Japanese Journal of Physical Fitness and Sports Medicine, 52(4), 359-367.

Noh, Y-E., Morris, T. \& Andersen, M. B. (2005). Psychosocial factors and ballet injuries. International Journal of Sport and Exercise Psychology, 3(1), 79-90.

Olmedilla A. (2005). Factores psicológicos y vulnerabilidad a las lesiones deportivas: Un estudio correlacional. Murcia: Quaderna.

Olmedilla, A., Andreu, M. D., Ortín, F. J. \& Blas, A. (2009). Ansiedad competitiva, percepción de éxito y lesiones: un estudio en futbolistas. Revista Internacional de Medicina y Ciencias de la Actividad Física y el Deporte, 9(33), 51-66.

Ortín, F.J. (2008). Factores psicológicos y sociodeportivos y lesiones en jugadores de fútbol semiprofesionales y profesionales (Tesis Doctoral). Universidad de Murcia, Murcia.

Passer, M. W. \& Seese, M. D. (1983). Life-stress and athletic injury: Examination of positive and negative events and three moderator variables. Journal of Human Stress, 9, 11-16.

Patterson, E. L., Smith, R. E., Everett, J. J. \& Ptacek J. T. (1998). Psychosocial factors as predictors of ballet injuries: interactive effects of life stress and social support. Journal of Sport Behavior, 21 (1), 101-112.

Petrie, T. A. (1992). Psychosocial antecedents of athletic injury: The effects of life-stress and social support on female collegiate gymnasts. Behavioral Medicine, 18, 127-138.

Petrie, T. A. (1993). Coping skills, competitive trait anxiety, and playing status: Moderating effects on the life stress-injury relationship. Journal of Sport and Exercise Psychology, 15, 261-274.

Rae, K. \& Orchard, J. (2007). The Orchard Sports Injury Classification System (OSICS) Version 10. Clinical Journal of Sport Medicine, 17(3), 201-204.

Rizopoulos, D. (2006). 1tm: an R package for latent variable modelling and item response theory analyses. Journal of Statistical Software, 17 (5), 1-25. 
Rubio, V. J., Pujals, C., de la Vega, R., Aguado, D. y Hernández, J. M. (2014). Autoeficacia y lesiones deportivas: ¿factor protector o de riesgo? Revista de Psicología del Deporte, 23(2), 439-444.

Smith, R. E., Ptacek, J. T. \& Patterson, E. (2000). Moderator effects of cognitive and somatic trait anxiety on the relation between life stress and physical injuries. Anxiety, Stress and Coping, 13(3), 269-288.

Smith, R. E., Smoll, F. L. \& Ptacek, J. T. (1990). Conjunctive moderator variables in vulnerability and resiliency research: Life-stress, social support and coping skills and adolescent sport injuries. Journal of Personality and Social Psychology, 58(2), 360-370.

Smith, R. E., Smoll, F. L. \& Wiechman, S. A. (1998). Measurement of trait anxiety in sport. En J.L. Duda (Ed.), Advances in Sport and Exercise Psychology Measurement. Morgantown: Fitness Information Technology.

Vealey, R. S. (2009). Confidence in sport. En B.W. Brewer (Ed.), Sport Psychology. Oxford: John Wiley \& Sons.

Vealey, R. S., Hayashi, S. W., Garner-Holman, M. \& Giacobbi, P. (1998). Sources of sport-confidence: Conceptualization and instrument development. Journal of Sport \& Exercise Psychology, 20, 54-80.

Wiese-Bjornstal, D. M. (2009). Sport injury and college athlete health across the lifespan. Journal of Intercollegiate Sports, 2, 64-80.

Williams, J. M. \& Andersen, M. B. (1998). Psychosocial antecedents of sport injury: review and critique of the stress and injury model. Journal of Applied Sport Psychology, 10, 5-25.

Williams, J. M. \& Andersen, M. B. (2007). Psychosocial antecedents of sport injury and interventions for risk reduction. En G. Tenenbaum y R.C. Eklund, (Eds.), Handbook of Sport Psychology. Nueva York: John Wiley \& Sons.

Wittig, A. F. \& Schurr, K. T. (1994). Psychological characteristics of women volleyball players: Relationships with injuries, rehabilitation and team success. Personality and Social Psychology Bulletin, 20(3), 322-330.

Recibido 25 de mayo de 2015

Revisión recibida 29 de julio de 2015

Aceptado 07 de diciembre de 2015

\section{Reseña de los autores}

Rosendo Berengüí Gil es Doctor en Psicología y profesor de la UCAM-Universidad Católica de Murcia, donde imparte docencia en los grados de Psicología y Educación. Cuenta con años de experiencia en el terreno aplicado como psicólogo de numerosos deportistas y entidades. En el ámbito de investigación, su línea de trabajo son los problemas asociados a la práctica deportiva, principalmente el análisis sobre el rol de las variables psicológicas en la prevención de lesiones deportivas y en el curso de la recuperación.

Jorge López Puga es Doctor en Psicología y Profesor en el Departamento de Ciencias de la Salud de la UCAM-Universidad Católica de Murcia. Desde 2005 a 2012, fue Profesor Asociado del Área de Metodología de las Ciencias del Comportamiento en el Departamento de Ciencias Humanas y Sociales de la Universidad de Almería. Su principal línea de investigación está vinculada a las redes bayesianas como herramientas de modelado estadístico. En el ámbito psicosocial, está interesado en las actitudes emprendedoras entre los estudiantes universitarios, especialmente con aquellas relacionadas con el emprendimiento sostenible.

Publicado en línea: 16 de diciembre de 2015 